\title{
正交实验设计优化离子聚合物金属复合材料的 力输出性能
}

\author{
何青松 ${ }^{(1) \dagger}$ ，于敏 ${ }^{(1 * \dagger}$ ，丁燕 ${ }^{(1)}$ 郭东杰 ${ }^{(1)}$, 李佳波 ${ }^{(1)}$, 戴振东 ${ }^{(1)}$ \\ (1) 南京航空航天大学仿生结构与材料防护研究所, 南京 210016; \\ (2) 南京航空航天大学机电学院, 南京 210016 \\ $\dagger$ 同等贡献 \\ *联系人, E-mail: yumin@nuaa.edu.cn
}

2011-01-04 收稿, 2011-04-12 接受

国家自然科学基金资助项目(50705043, 50805076, 50975140)

\begin{abstract}
摘要 离子聚合物金属复合材料(ionic polymer metal composite)作为新型驱动材料在微小系统 驱动等方面有着明确的应用前景, 输出力较低和对水环境的依赖限制了该材料进一步的应用. 本文采用正交实验设计法, 针对 IPMC 制备过程中的 3 个主要参数, 选用 $\mathrm{L}_{16}\left(4^{5}\right)$ 正交表, 以输 出力为指标开展制备工艺的优化. 结果表明主镀过程铂氨复合物的浓度对 IPMC 输出力的影响 最大, Nafion 膜中四乙氧基硅(TEOS)含量对输出力的影响次之, 还原剂浓度对输出力没有显著 影响. 在实验确定的最优条件下, IPMC 的最大输出力达到 $50 \mathrm{mN}$, 相应的最大位移为 $14 \mathrm{~mm}$. 在最大输出位移性能相当的情况下, 较常规条件最大力输出提高了 2.4 倍, 有效工作寿命大幅 提高, 有效力输出延长了5.8倍, 有效位移输出性能延长了 5 倍. 该研究为 IPMC 进一步的应用 奠定了坚实的基础.
\end{abstract}

驱动技术是机械系统进步的重要标志. 从蒸汽 机、内燃机到电动机的技术革新, 使机械系统的灵活 性、能量利用效率和使用方便性得到了一次次飞跃和 提升 ${ }^{[1]}$. 微机电系统发展呼唤新型驱动材料和驱动部 件的发明和创新 ${ }^{[2]}$, 已成为未来材料、机械和电气工 程交叉领域迫切需要解决的关键问题之一. 基于压 电效应和相变效应的驱动材料和技术已有不少研 究 $^{[3]}$, 但因压电材料驱动电压高、位移小, 相变材料 适应频率低、能耗大, 还难于满足微型机器人、微机 电系统对高灵活性、高几余度和高负载/体重比的要 求, 具有肌肉驱动特性的 “人工肌肉” 以其低电压驱 动、大变形等优点受到了各界的高度重视 ${ }^{[4,5]}$.

离子聚合物金属复合材料(ionic polymer metal composite) 是一种新型的电活性聚合物材料(EAP), 又被称为 “人工肌肉”, 它是在全氟磺酸离子聚合物
(如 Nafion 膜)的基材表面沉积铂、金等贵金属而获得 的有机-无机复合材料 ${ }^{[6-8]}$. IPMC 在低电压激励下能 产生超过 $90^{\circ}$ 的弯曲变形, 其电致动变形机制源于阳 离子的电泳现象，在外加电场的作用下，基材中的阳 离子与水分子结合在一起迁移到阴极, 导致阳极含 水量减少, 阴极含水量增加, 使 IPMC 变形 ${ }^{[9 ~ 11]}$. 和 压电陶瓷、形状记忆合金、磁致伸缩材料等驱动材料 的性能相比, IPMC 具有质量轻、驱动电压低、位移 大、无噪音、驱动能量密度较高等优点 ${ }^{[12,13]}$, 在微器 件驱动(如体内检测机器人、光机电系统、微型机器 人等)方面有着巨大的应用潜力 ${ }^{[14]}$. 使用 IPMC 驱动 器, Shahinpoor 等人 ${ }^{[10]}$ 研制了微型四爪抓取器; Zhang 等人 ${ }^{[15]}$ 研制了能实现泳动、行走和漂浮的水下机器 人; Yeom 等人 ${ }^{[16]}$ 研制了仿水母机器人. 输出力较低 (仅毫牛级 $)^{[6,14]}$ 限制了 IPMC 的进一步应用, 因此不

英文版见: He Q S, Yu M, Ding Y, et al. Force optimization of ionic polymer metal composite actua-tors by an orthogonal array method. Chinese Sci Bull, 2011, 56, doi: 10.1007/s11434-011-4509-9 
少研究致力于通过优化制备工艺来提高 IPMC 的输 出力. Kim 等人 ${ }^{[17]}$ 用聚合物溶液浇铸技术获得了厚的 全氟磺酸膜, 以提高 IPMC 的输出力, 当输人电压为 $6 \mathrm{~V} \mathrm{DC}$ 时, 厚度为 $2 \mathrm{~mm}$ 的 IPMC 的输出力达到了 20 gf. Onishi 等人 ${ }^{[18]}$ 采用 8 次化学镀生成碎片状金属电 极(厚度约 $20 \mu \mathrm{m}$ )来改进 IPMC 的弯曲性能. Lee 等 人 ${ }^{[19]}$ 通过热压 Nafion 膜和增加化学镀的次数 (4 次) 来提高 IPMC 的力输出性能, 热压 5 片 Nafion 膜后制 备的 IPMC 的最大输出力约为 $4 \mathrm{gf}$. Lee 等人 ${ }^{[20]}$ 使用 碳纳米管作为添加剂制备高性能的 IPMC, 最大输出 应力为 $0.236 \mathrm{gf} / \mathrm{mm}^{2}$.

IPMC 制备过程涉及因素多, 且存在复杂的交互 作用, 制备条件的精细控制对 IPMC 的性能有显著影 响. 目前各研究单位的制备工艺不尽相同, 存在较大 差异, 尚需进一步优化. 本课题组前期采用溶胶凝胶 反应研制了无定形氧化硅掺杂的全氟磺酸薄膜, 以 提高 IPMC 的输出力和有效非水工作时间 ${ }^{[21]}$. 在前期 IPMC 研究的积累上, 本文总结影响 IPMC 性能的因 素，采用正交实验设计法，优化 IPMC 的制备工艺， 以提高 IPMC 复合材料的力输出性能.

\section{1 材料和方法}

(i) 实验材料. Nafion DE520( 5\%) 溶液从 美国 Dupont 公司购买. 硅橡胶(PDMS, Sylgad184)从 美国 Dow Corning 公司购买. TEOS, $\mathrm{Pt}\left(\mathrm{NH}_{3}\right)_{4} \mathrm{Cl}_{2}$, $\mathrm{NH}_{4} \mathrm{OH}, \mathrm{NaBH}_{4}, \mathrm{H}_{2} \mathrm{NNH}_{2}$ 和 $\mathrm{H}_{2} \mathrm{NOH} \cdot \mathrm{HCl}$ 从 SigmaAldrich 公司购买.

(ii) IPMC 的制备. 前期采用化学镀的方法成 功制备了 IPMC 复合材料 ${ }^{[14,21]}$, 主要制备过程包括: (1) 浇铸氧化硅掺杂的 Nafion 膜: 按一定比例将 Nafion 溶液, 四乙氧基硅 (TEOS) 溶液依次加人 PDMS 模具中, 充分摚拌后将模具置人真空烤箱中, 温度设置为 $70^{\circ} \mathrm{C}$, 成膜后在 $150^{\circ} \mathrm{C}$ 下保持 $8 \mathrm{~min}$ 退火 得到 Nafion 膜. TEOS 含量分别为: $0 \%, 0.5 \%, 1 \%$, $1.5 \%$, 所得膜厚分别为: $0.22,0.28,0.34,0.42 \mathrm{~mm}$. (2) Nafion 膜表面织构化: 对 Nafion 膜进行直线式研磨, 得到了各向异性的表面织构. (3) 离子吸附 ${ }^{[22]}$ : 该步 骤中铂氨复合物的 $\left[\mathrm{Pt}\left(\mathrm{NH}_{3}\right)_{4}\right]^{2+}$ 与 Nafion 膜内的 $\mathrm{H}^{+}$发 生交换, 使得 $\left[\mathrm{Pt}\left(\mathrm{NH}_{3}\right)_{4}\right]^{2+}$ 离子吸附在 Nafion 膜上, 铂 氨复合物的浓度对 IPMC 金属镀层有着重要的影响. （4）主化学镀：使用 $\mathrm{NaBH}_{4}$ 作为还原剂, 将吸附在 Nafion 膜上的 $\mathrm{Pt}$ 离子还原成纳米级的 $\mathrm{Pt}$ 金属颗粒,
沉积在 Nafion 膜的表面 ${ }^{[23]}$. (5) 次化学镀：次化学镀 是在主化学镀沉积的金属电极上继续沉积 Pt 颗粒以 减少表面电阻. (6) 离子交换: 在 $30^{\circ} \mathrm{C}$ 下将 IPMC 试 样浸泡于 $1.5 \mathrm{~N} \mathrm{LiCl}$ 溶液中 $24 \mathrm{~h}$, 以交换锂离子.

(iii) 优化方案设计. IPMC 制备过程中涉及因 素多, 在前期 IPMC 研究的积累上, 总结影响 IPMC 性能的因素, 确定 3 个对 IPMC 制备过程影响可能最 大的因素：主镀还原剂浓度，主镀铂氨复合物浓度， Nafion 膜中 TEOS 含量. 采用正交实验设计法, 研究 IPMC 制备过程中上述 3 因素对 IPMC 制备工艺的影 响, 以提高 IPMC 复合材料的力输出和离水工作寿命. 正交实验设计法可以大大减少实验次数来达到对目 标参数的优化. 实验中各因子设定 4 个水平(低, 中, 较高, 高)(表 1), 选用 $\mathrm{L}_{16}\left(4^{5}\right)$ 正交表来设计实验方案 (表 2)，表 2 中实验号对应制备的 16 种试样，第 1, 2, 3 列分别代表因子 A, B, C, 第 4列和第 5 列用于估算误 差 $e, G_{i}$ 为对应第 $i$ 个实验得到的 IPMC 试样的最大输 出力, $S_{j}$ 为各因子的偏差平方和, $I_{j}, I I_{j}, I I I_{j}, I V_{j}$ 分别为 第 $j$ 列“低” “中” “较高” “高”水平对应的 $G_{i}$ 数据之和. 对于正交表中误差的偏差平方和，可以用正交表中 未安排因子的空白列的偏差平方和来计算, 所以它 们的偏差平方和中不包括因子水平间的差异，而仅 仅反应了实验误差的大小.

(iv) SEM 观测. 用 Schottky 场扫描电子显微镜 (LEO 1530VP) 来观察薄膜的形貌. 断面试样通过低 温冷断制备, 将 Nafion 膜和 IPMC 试样浸泡于液氮中 $0.5 \mathrm{~h}$ 后断开. 在观测前所有试样表面均沉积了直 径 $20 \mathrm{~nm}$ 铂颗粒.

(v) IPMC 驱动性能测试. 图 1 为 IPMC 驱动性 能测试系统. 由驱动信号生成单元、位移传感器和力 传感器组成. 信号发生单元采用数字合成低频功率 信号发生器(SP1651, 盛普科技)。位移传感器为基恩 士的激光位移传感器(LK-G80, KEYENCE), 测试时 要求激光光束垂直于 IPMC 初始位置的表面, 测点距 试样固定端 $16 \mathrm{~mm}$. 力传感器采用 UMT-2 微摩擦试

表 1 因子和对应的水平

\begin{tabular}{ccccc}
\hline 因子 & 1 (低) & 2(中) & 3(较高) & 4 (高) \\
\hline $\begin{array}{c}\text { 还原剂的浓度 } \mathrm{A}(\%) \\
\begin{array}{c}\text { 主镀铂氨复合物的浓度 } \\
\mathrm{B}(\mathrm{mg} / \mathrm{mL})\end{array}\end{array}$ & 2 & 2.5 & 2.5 & 3.5 \\
$\begin{array}{c}\text { Nafion 膜中 TEOS 含量 } \\
\mathrm{C}(\%)\end{array}$ & 0 & 0.5 & 1 & 3.5 \\
\hline
\end{tabular}


表 2 正交实验设计表

\begin{tabular}{ccccccc}
\hline 实验号 & $1(\mathrm{~A})$ & $2(\mathrm{~B})$ & $3(\mathrm{C})$ & $4(e)$ & $5(e)$ & $G_{i}(\mathrm{mN})$ \\
\hline 1 & 1 & 1 & 1 & 1 & 1 & 11.96 \\
2 & 1 & 2 & 2 & 2 & 2 & 25.97 \\
3 & 1 & 3 & 3 & 3 & 3 & 19.99 \\
4 & 1 & 4 & 4 & 4 & 4 & 32.05 \\
5 & 2 & 1 & 2 & 3 & 4 & 21.95 \\
6 & 2 & 2 & 1 & 4 & 3 & 19.99 \\
7 & 2 & 3 & 4 & 1 & 2 & 37.04 \\
8 & 2 & 4 & 3 & 2 & 1 & 35.97 \\
9 & 3 & 1 & 3 & 4 & 2 & 19.99 \\
10 & 3 & 2 & 4 & 3 & 1 & 32.05 \\
11 & 3 & 3 & 1 & 2 & 4 & 24.01 \\
12 & 3 & 4 & 2 & 1 & 3 & 33.03 \\
13 & 4 & 1 & 4 & 2 & 3 & 19.99 \\
14 & 4 & 2 & 3 & 1 & 4 & 25.97 \\
15 & 4 & 3 & 2 & 4 & 1 & 37.04 \\
16 & 4 & 4 & 1 & 3 & 2 & 28.03 \\
$I_{j}$ & 89.97 & 73.89 & 83.99 & 108 & 117.02 & \\
$I I_{j}$ & 114.95 & 103.98 & 117.99 & 105.94 & 111.03 & \\
$I I I_{j}$ & 109.08 & 118.08 & 101.92 & 102.02 & 93.00 & \\
$I V_{j}$ & 111.03 & 129.08 & 121.13 & 109.07 & 103.98 & \\
$R_{j}{ }^{2}$ & 45534.21 & 12117.44 & 46036.12 & 45191.63 & 45482.18 & \\
$S_{j}=R_{j}{ }^{2} / 4$ & 92.90 & 428.37 & 218.37 & 7.25 & 79.89 & \\
$-C T$ & 9 & & & & & \\
\hline & & & & &
\end{tabular}

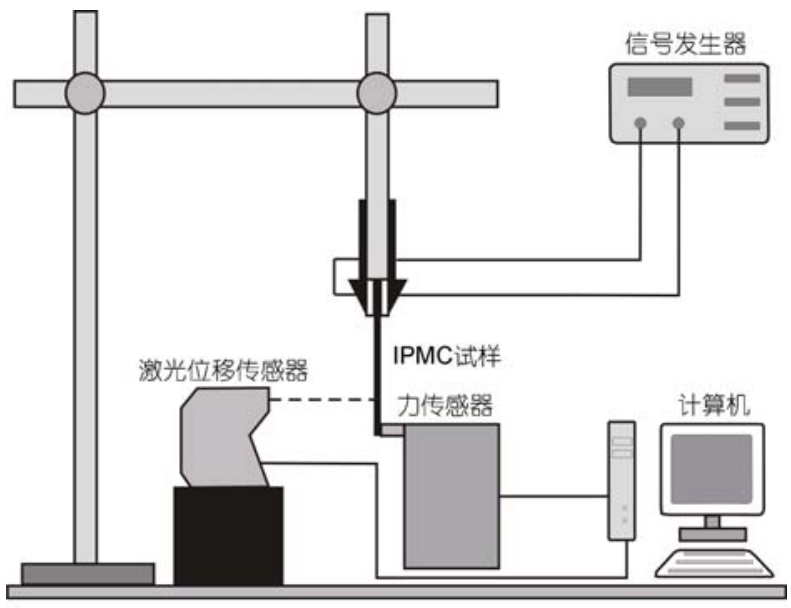

图 1 IPMC 驱动性能测试平台

验机 (CETR) 的量程为 $10 \mathrm{~g}$ 的微传感器, 测点在 IPMC 的末端. 实验中 IPMC 剪裁成长宽为 $20 \mathrm{~mm} \times 5 \mathrm{~mm}$ 的条形试样. 测试时间持续 $800 \mathrm{~s}$. 测试中驱动信号 为 $0.1 \mathrm{~Hz}, 3.0 \mathrm{~V}$ 的正弦信号, 测试前试样充分润湿, 实验在空气中完成. 在测试过程中同时采集了 IPMC 的侧向力 (平行于试样)和垂直力 (垂直于试样), 但由
于侧向力很小, 本文用垂直力表征 IPMC 的力输出性 能. 实验中通过传感器测头限制 IPMC一侧位移, 获 得单方向的力数据.

\section{2 结果和分析}

\section{1 方差分析}

对 16 组实验号对应的 IPMC 试样, 在测试平台 上测试了每个试样对应的最大输出力和最大输出位 移(图 2). 结果显示 IPMC 试样的最大输出力界于 $11.96 \sim 37.04 \mathrm{mN}$ 之间，最大位移值界于 3.60 13.96 $\mathrm{mm}$. 根据所测试的最大输出力数据 $G_{i}$, 可计算出因 子 $\mathrm{A}, \mathrm{B}, \mathrm{C}$ 及误差 $e$ 的偏差平方和 $S_{\mathrm{A}}=92.90, S_{\mathrm{B}}=$ $428.37, S_{\mathrm{C}}=218.37, S_{e}=87.14$. 总的自由度 $f_{\text {总 }}=n-1$ $=15$ ( $n$ 为实验次数), 因子 $\mathrm{A}, \mathrm{B}, \mathrm{C}$ 的偏差平方和的自 由度为 $f_{\mathrm{A}}=f_{\mathrm{B}}=f_{\mathrm{C}}=3$ (因子的水平数 -1 ), 误差 $e$ 的偏 差平方和的自由度 $f_{e}=f_{\text {总 }}-f_{\mathrm{A}}-f_{\mathrm{B}}-f_{\mathrm{C}}=6$. 由因子 水平的改变引起的平均偏差平方和与误差的平均偏 差平方和的比值可以得出各因子的 $F$ 比: $F_{\mathrm{A}}=2.13$, $F_{\mathrm{B}}=9.83, F_{\mathrm{C}}=5.01$. 因子对实验结果影响的显著度 可由因子的 $F$ 比与临界值的比较获得. $F$ 比大于临界 值, 可以认为实验结果的差异主要是由因子水平的 改变引起的; $F$ 比小于临界值, 说明实验结果的差异 主要是由实验误差引起的. 临界值根据统计数学原 理编制的 $F$ 分布表确定，依据 $F$ 检验的临界值表查得： 信度 $0.10: F_{0.10}(3,6)=3.29$; 信度 $0.05: F_{0.05}(3,6)=$ 4.76; 信度 $0.01: F_{0.01}(3,6)=9.78$. 对于 A 因子, $F_{\mathrm{A}}<$ $F_{0.10}(3,6)$, 说明还原剂浓度的变化对 IPMC 输出力没 有显著的影响; 对于 B 因子, $F_{\mathrm{B}}>F_{0.01}(3,6)$, 即主镀 铂氨复合物浓度的变化对 IPMC 输出力有高度显著的 影响; 对于 $\mathrm{C}$ 因子, $F_{0.05}(3,6)<F_{\mathrm{C}}<F_{0.01}(3,6)$, 说明 TEOS 含量的改变对 IPMC 输出力有显著的影响.

表 3 给出了本次正交实验方差分析的结果, 根据

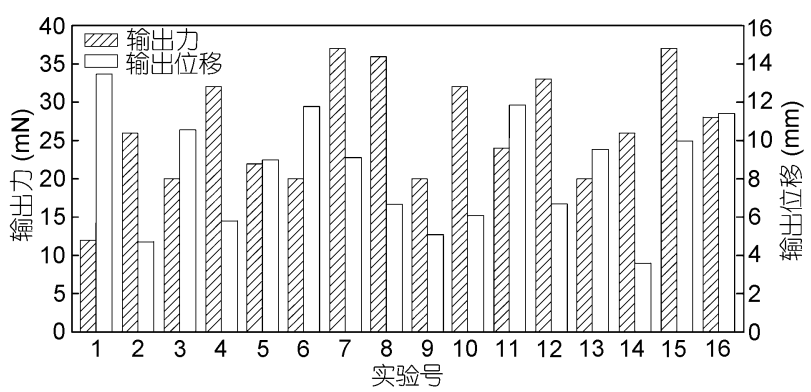

图 2 正交实验各实验号对应最大输出力和最大输出位移 
表 3 方差分析 ${ }^{\text {a) }}$

\begin{tabular}{crcccc}
\hline 方差来源 & $\begin{array}{c}\text { 偏差 } \\
\text { 平方和 }\end{array}$ & 自由度 & $\begin{array}{c}\text { 平均偏差 } \\
\text { 平方和 }\end{array}$ & $F$ 比 & 显著性 \\
\hline 还原剂浓度 $(\mathrm{A})$ & 92.90 & 3 & 30.97 & 2.13 & $/$ \\
铂氨浓度 $(\mathrm{B})$ & 428.37 & 3 & 142.79 & 9.83 & $* *$ \\
$\mathrm{TEOS}$ 含量 $(\mathrm{C})$ & 218.37 & 3 & 72.79 & 5.01 & $*$ \\
误差 $(e)$ & 87.14 & 6 & 14.52 & & \\
\hline
\end{tabular}

a)/代表没有显著的影响, **代表高度显著的影响, *代表显著 的影响

表 2 中各水平对应的输出力之和 $I_{j}, I I_{j}, I I I_{j}, I V_{j}$ 的大小 可以看出选取 $\mathrm{A}$ 因子的 2 水平, $\mathrm{B}$ 因子的 4 水平, $\mathrm{C}$ 因子的 4 水平时值最大, 因此通过方差分析的结果最 终确定本实验的最优条件为 $\mathrm{A}_{2} \mathrm{~B}_{4} \mathrm{C}_{4}$, 即主镀还原剂 浓度为 $1.5 \%$, 主镀铂氨复合物浓度为 $3.5 \mathrm{mg} / \mathrm{mL}$, Nafion 膜的 TEOS 含量为 $1.5 \%$.

\section{2 力和位移输出}

本文在常规条件 $\left(\mathrm{A}_{2} \mathrm{~B}_{1} \mathrm{C}_{1}\right)$ 和最优条件 $\left(\mathrm{A}_{2} \mathrm{~B}_{4} \mathrm{C}_{4}\right)$ 下 采用化学镀的方法制备了 IPMC 复合材料, 并测试了 该材料在工作时间持续 $800 \mathrm{~s}$ 内的输出性能, 测试结 果如图 3 所示. 图 3(b)和 3(d)为常规条件和优化条件 下 IPMC 的力和位移响应, 在图 3(b)和 3(d)中提取正 弦电压每一周期的最大力输出和位移输出, 汇总得 到如图 3(a)和 3(c) 所示最大输出力及最大输出位移随 时间的变化. 结果得到常规条件下制备的 IPMC 的力 最大值为 $14.80 \mathrm{mN}$ ，优化条件下力最大值为 49.98 $\mathrm{mN}$, 最大输出力增大了 2.4 倍. 常规制备条件下 IPMC 试样的位移最大值 $13.96 \mathrm{~mm}$, 优化条件下位移 最大值为 $14.10 \mathrm{~mm}$, 可见优化后位移输出并未受到 影响. 因此在最大输出位移相当的基础上, 优化后的 IPMC 的最大力输出有了大幅提升. 而且优化后的 IPMC 的最大输出力 $(49.98 \mathrm{mN})$ 和最大输出位移 (14.10 mm)均高于本次正交实验对应的 16 种试样的 最大输出力 $(11.96 \sim 37.04 \mathrm{mN})$ 和最大输出位移 $(3.60$ $\sim 13.96 \mathrm{~mm}$ ).

基于 IPMC 复合材料的驱动机理，离水工作一定 时间后其输出性能会逐渐衰退. 通过测试输出力和 输出位移随时间的变化关系来研究两种条件下 IPMC 的稳定工作性能. 其稳定工作性能可用有效工作寿 命来表征, 本文规定输出性能达到最大值的 $80 \%$ 所 经历的时间为其有效工作寿命. 从图 3(a)和 3(c)还可 以看到, 优化后 IPMC 试样的稳定工作性能得到了提 升. 常规制备条件下的 IPMC 试样输出力在 $38 \mathrm{~s}$ 达到
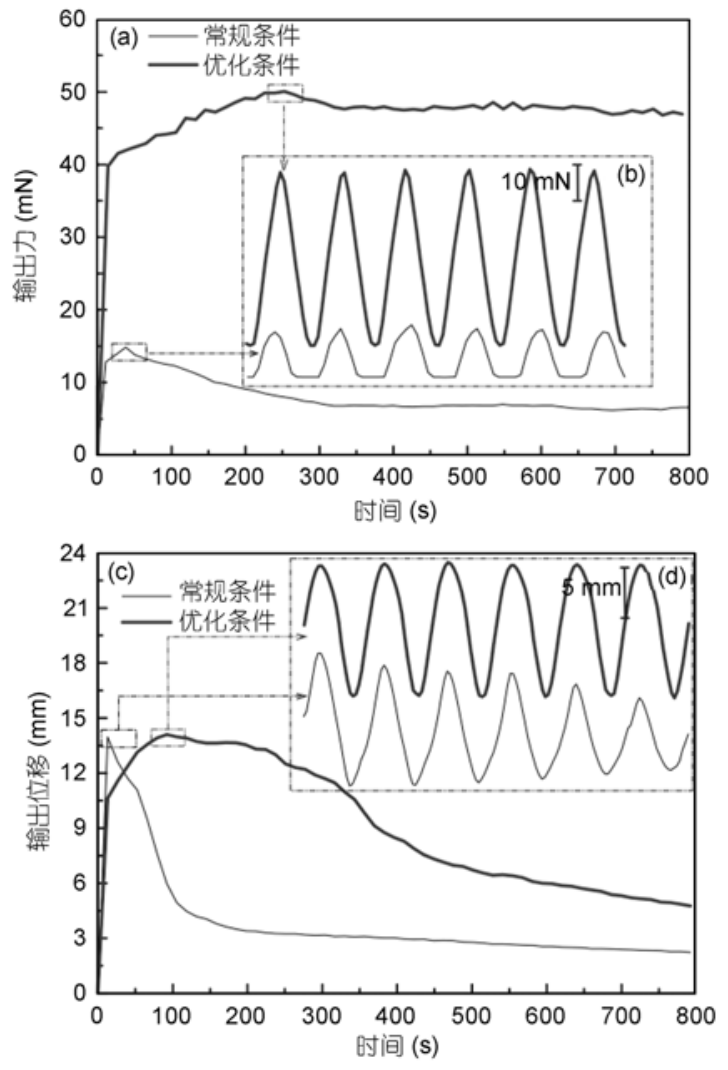

图 3 常规制备和优化制备两种条件下输出力和输出位移 随时间变化关系的比较

(a) 最大输出力; (b) 力输出-时间响应; (c) 最大输出位移; (d) 位移 输出-时间响应

最大值 $(14.80 \mathrm{mN})$, 之后就逐渐减小, 在 $118 \mathrm{~s}$ 达到 最大值的 $80 \%(11.76 \mathrm{mN})$, 在 $800 \mathrm{~s}$ 时刻衰减至 6.57 $\mathrm{mN}$, 衰减了约 $60 \%$; 而优化制备条件下的 IPMC 试 样在一开始 $14 \mathrm{~s}$ 输出力即达到 $39.78 \mathrm{mN}$, 在 $252 \mathrm{~s}$ 达 到最大值为 $49.98 \mathrm{mN}$, 之后略有降低, 并保持该水 平一直到实验结束. 在 $800 \mathrm{~s}$ 时刻输出力为 $47.04 \mathrm{mN}$, 只衰减了 $6 \%$ 左右. 因此优化后 IPMC 的力输出性能 大幅提升, 衰减程度大大降低, 有效工作寿命提高了 5.8 倍. 同样地, 对于位移输出, 常规条件下制备的 IPMC 试样的输出位移在一开始达到最大值(13.96 $\mathrm{mm})$, 而后就立刻快速减小, 不到 $1 \min (52.8 \mathrm{~s})$ 衰减 至最大值的 $80 \%$ 左右(图 3(c)). 优化条件下制备的 IPMC 试样在工作 $5 \mathrm{~min}(316.8 \mathrm{~s})$ 后依然能保持最大值 的 $80 \%$ 左右，表明其在位移输出的稳定性方面也得 到了明显的改善, 有效工作寿命提高了 5 倍. 因此较 常规条件, 制备工艺优化后 IPMC 的离水有效工作寿 命大大延长, 有效力输出延长了约 $10 \mathrm{~min}$, 提高了 5.8 倍, 有效位移输出延长了约 $4 \mathrm{~min}$, 提高了 5 倍. 
图 4(a), 4(b)所示为常规与优化两种条件下输出 力和输出位移在测试时间内的最大值、平均值和最小 值的比较. 最大值反映了 IPMC 试样的最大工作能力, 平均值和最小值则反映了试样的稳定工作性能. 从 图 4 中可清楚直观地看到, 优化制备条件下不论是最 大工作能力还是稳定工作性能, 试样的力输出性能 都得到了大幅提升; 与此同时, 最大输出位移没有降 低, 而且位移输出的稳定性能得以加强.

\section{3 断面形貌分析}

针对方差分析结果确定的最优工艺条件, 下面 通过 SEM 观测分析了主镀铂氨复合物浓度和 TEOS 含量两个制备参数对 IPMC 力输出性能的影响.

选取主镀铂氨复合物浓度分别为 $2,2.5,3,3.5$ $\mathrm{mg} / \mathrm{mL}$ 对应的 IPMC 试样(对应实验号 1,2,3,4 的试 样)的断面进行电子显微镜测试(图 5). 从图 5 中可以 看出, 随着铂氨复合物浓度的提高, 金属铂层厚度逐 渐增加, 分别约为 $6,7,10,24 \mu \mathrm{m}$, 即 IPMC 的表面金 属电极厚度逐渐增加. IPMC 的表面铂层越厚, 电极
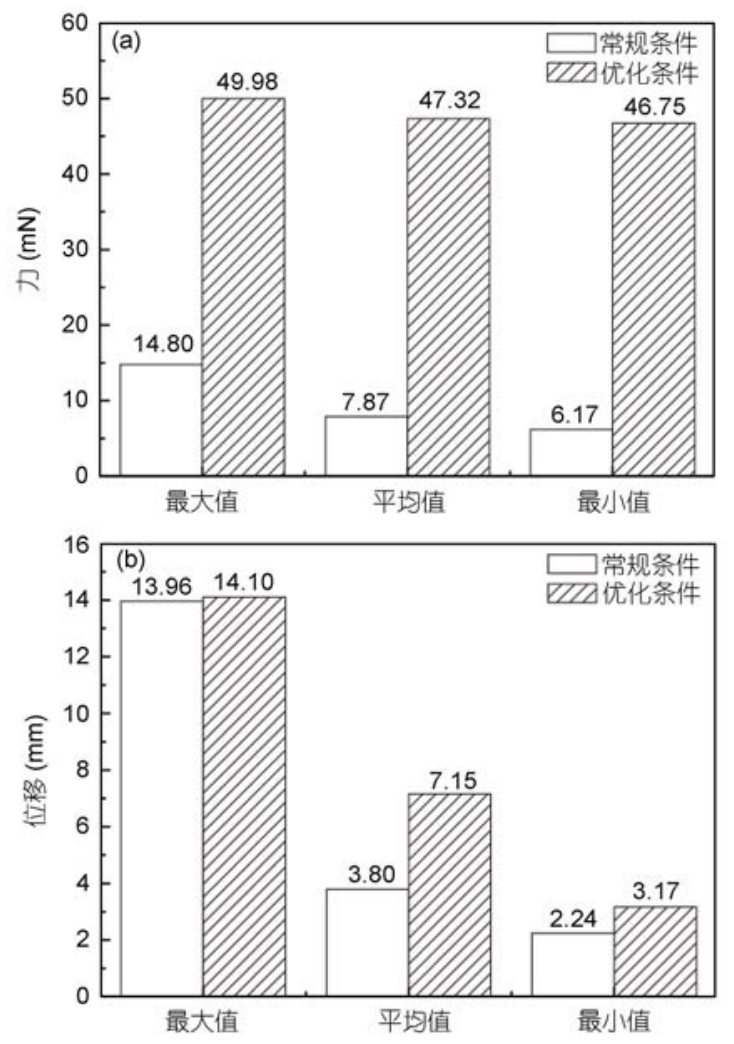

图 4 常规制备和优化制备两种条件下输出力和输出位移 的比较

(a) 输出力; (b) 输出位移
的表面电阻越小 ${ }^{[18,19]}$, 同样电压输人下电流则越大, 这将有利于提高 IPMC 的输出力. 当铂氨复合物浓度 为 $3.5 \mathrm{mg} / \mathrm{mL}$ 时，在金属铂层和全氟磺酸薄膜之间没 有观察到明显的分界面, 说明铂颗粒较深地嵌人到 全氟磺酸基体膜中, 金属铂层的厚度达到最大(约 24 $\mu \mathrm{m})$, 从而其 IPMC 的表面电阻最小, 输出力则最大. 从图 5(d)中还可观察到, 随着铂氨复合物浓度提高, 金属铂层与基体膜的过渡层明显加大，结合更紧密、 均匀, 可有效降低膜内水分子的泄漏蒸发, 有利于材 料的驱动性能. 基于化学镀理论, 随着铂盐浓度增 加沉积速度上升, 但铂盐浓度过高沉积速度反而下 降(镀液自分解), 同样的化学镀时间内镀层铂的含量 降低. 而且由于 Nafion 薄膜的离子交换能力有限, 可 吸附 $\left[\mathrm{Pt}\left(\mathrm{NH}_{3}\right)_{4}\right]^{2+}$ 离子是有限的, 因此主镀铂氨复合物 浓度亦不是越高越好。

图 6 所示为不同 TEOS 含量 Nafion 膜的断面 SEM 图. IPMC 基底膜的制备中添加 TEOS 是利用全 氟磺酸的酸性 ( $\mathrm{pH}$ 为 2.0 ), 二氧化硅前体(即 TEOS) 缓慢发生原位溶胶一凝胶反应，产生的二氧化硅与极 性的全氟磺酸大分子共结晶, 形成了一种有机-无机 杂化薄膜, 以改善 IPMC 基底膜的储水性能. 当 TEOS 含量分别为 $0.5 \%, 1 \%, 1.5 \%$ 时, Nafion 膜内生 成不同含量的无定形氧化硅和不同尺寸的微管道(图 6(b) (d)), 无定形的氧化硅具有较好的亲水性, 而膜 内存在的微管道可以储存水分子. 当不添加 TEOS 时, Nafion 膜的断面比较光滑, 较少有微管道呈现(图 $6(a))$, 当添加的 TEOS 含量分别为 $0.5 \%, 1 \%, 1.5 \%$ 时, Nafion 膜的断面均出现了清晰的微管道, 且随着 TEOS 含量的增加, 微管道的直径逐渐增加, 分别约 为 $1,5,10 \mu \mathrm{m}$, 如图 6(b) (d) 所示, 它们与 Nafion 薄 膜内部固有的纳米级管道相互连接, 共同构成了 IPMC 复合材料内部水合阳离子迁移的通道网络. 这 些微管道的增大将会改变 IPMC 的储水能力，储水能 力可用含水量来表征. 含水量 $w$ 可以表示为

$$
w=\frac{M_{\mathrm{wet}}-M_{\mathrm{dry}}}{M_{\mathrm{dry}}},
$$

其中 $M_{\text {wet }}$ 和 $M_{\mathrm{dry}}$ 为吸水和脱水状态下 Nafion 膜的质 量, 该质量分别由电子天平(METTLER TOLEDO, AL204)称得. 经计算可得当 TEOS 含量分别为 0 , $0.5 \%, 1 \%, 1.5 \%$ 时, Nafion 膜的含水量分别为 $1.14 \%$, $4.09 \%, 6.53 \%, 15.27 \%$ (图 7), 该结果表明, 与空白膜 相比, 含水量提高了 2.6 12.4 倍, 且含水量随 TEOS 

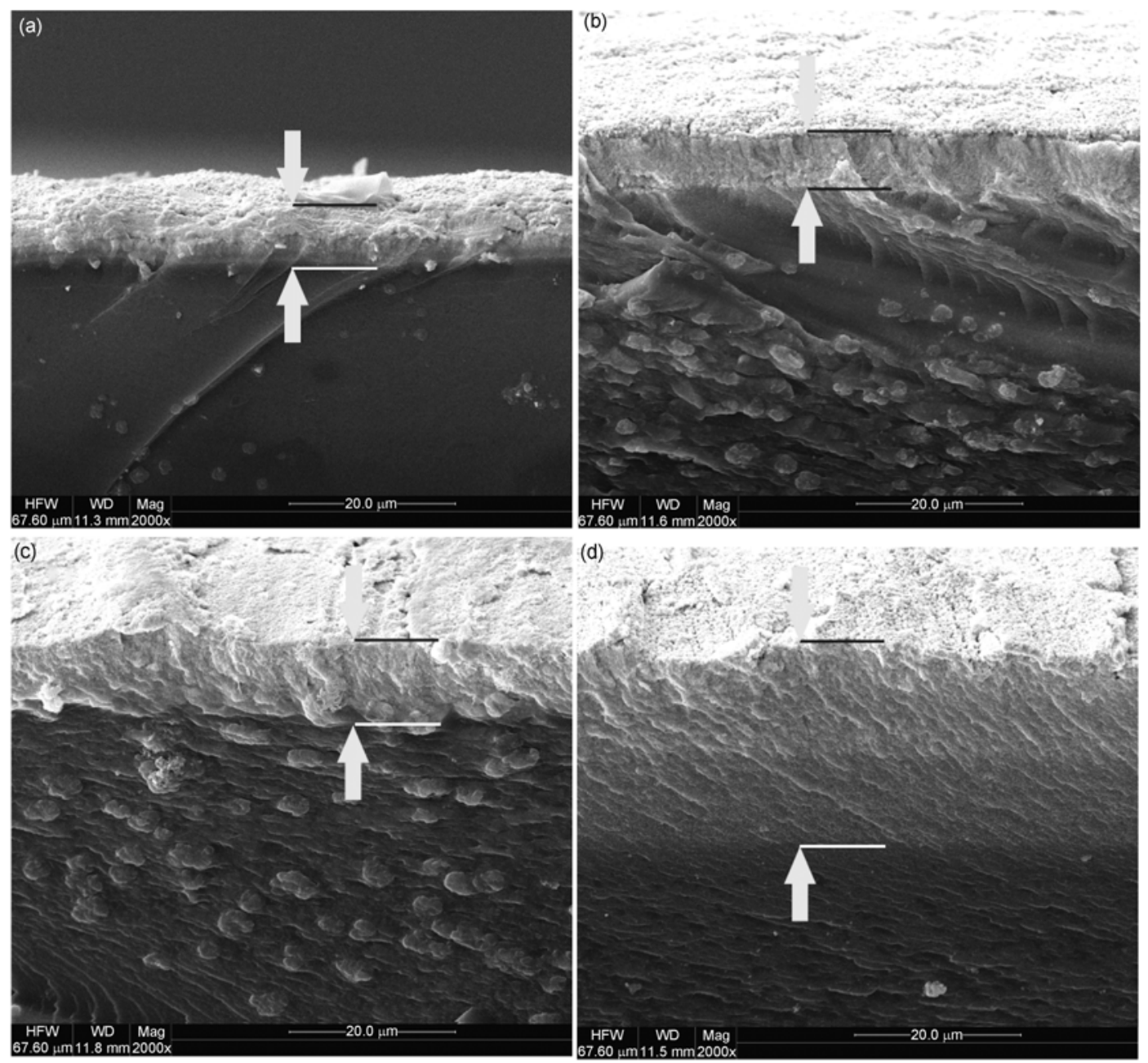

(d)

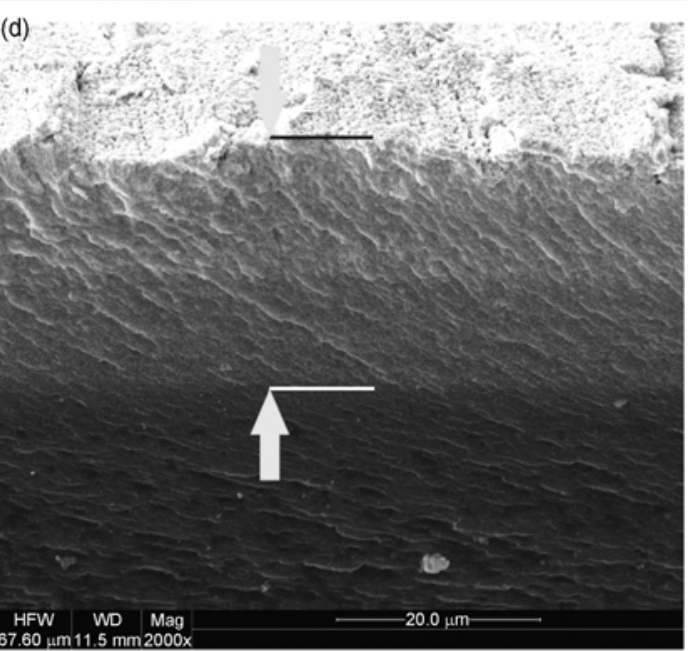

图 5 不同铂氨复合物浓度制备的 IPMC 试样断面 SEM 图 (a) $2 \mathrm{mg} / \mathrm{mL}$; (b) $2.5 \mathrm{mg} / \mathrm{mL}$; (c) $3 \mathrm{mg} / \mathrm{mL}$; (d) $3.5 \mathrm{mg} / \mathrm{mL}$

含量的增加而增加. 由于 TEOS 含量的增加, 膜内微 管道的直径增加, 膜的含水量提高, 同等电信号输人 条件下参与迁移的水合阳离子大幅提高, 从而制备 出储水能力增强的全氟磺酸薄膜. 当 TEOS 的含量为 $1.5 \%$ 时, Nafion 膜内的微管道直径 $(10 \mu \mathrm{m})$ 和含水量 $(15.27 \%)$ 为最大, 在外加电场作用下其参与迁移的水 合阳离子最多, 为提高 IPMC 的力和位移输出性能、 增加离水工作寿命提供保证.

综上所述, 制备过程中 B 因子的 4 水平(铂氨复 合物溶液浓度 $3.5 \mathrm{mg} / \mathrm{mL}$ ) 和 C 因子的 4 水平(Nafion 膜中 TEOS 含量 $1.5 \%$ ) 对提高 IPMC 的输出性能有着 显著的作用. 合适的铂氨复合物浓度有利于形成致 密的较厚的表面金属电极, 降低了表面电阻, 减缓水 分子的泄漏. 添加 TEOS 后全氟磺酸薄膜内形成了具
有不同尺寸的微管道, 增加了 Nafion 基底膜的含水 量, 进而影响 IPMC 的最大输出力、最大输出位移和 稳定工作性能. 优化后 IPMC 的最大输出力提高了 2.4 倍, 力输出的有效工作寿命延长了 5.8 倍, 位移输 出的有效工作寿命延长了 5 倍(图 3). 因此 IPMC 制 备过程的精细控制对 IPMC 的驱动性能有着关键的 作用.

\section{3 讨论}

IPMC 复合材料在低电压驱动下能产生大的位移, 但其输出力却相对较小. 如何提高输出力就成为 IPMC 驱动器应用的一个关键和难点. 结合上述实验 结果与分析, 从膜的制备与变形机理人手, 下面从 3 个方面进行比较讨论. 


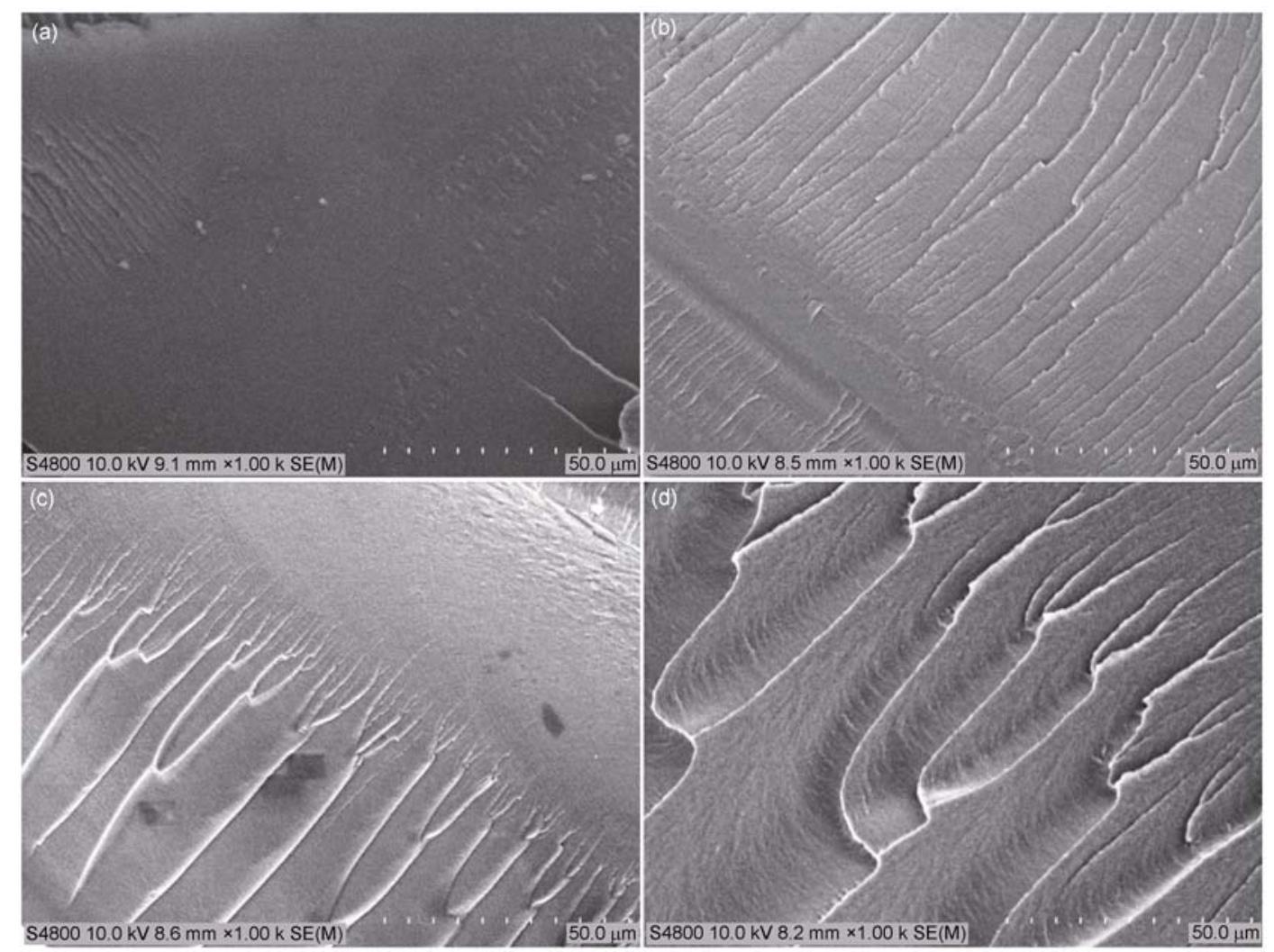

图 6 不同 TEOS 含量 IPMC 基底 Nafion 膜断面 SEM 图

(a) 0 ; (b) $0.5 \%$; (c) $1 \%$; (d) $1.5 \%$

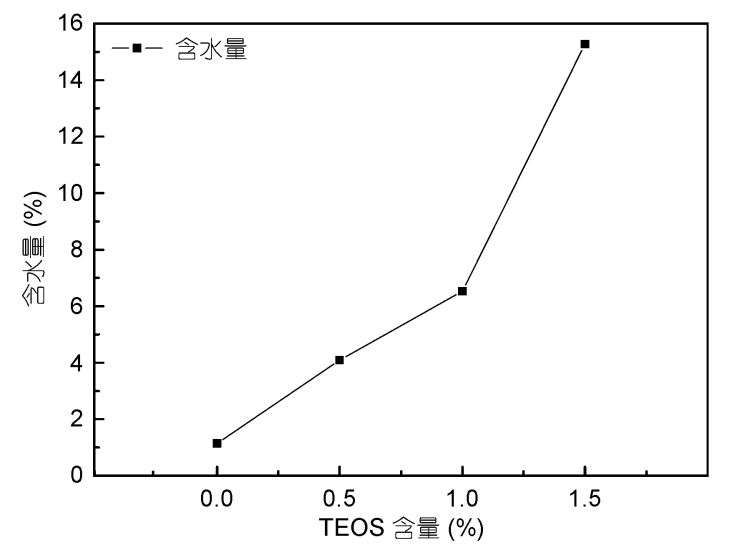

图 7 不同 TEOS 含量 IPMC 基底 Nafion 膜的含水量 比较

\section{1 优良的金属电极}

IPMC 存在表面电阻, 加在电极表面的电场沿着 长度方向会产生一定的电压梯度 ${ }^{[8]}$, 且随表面电阻的 增大而增大 ${ }^{[7]}$. 所以 IPMC 内部水合阳离子所受电场 力是不均匀的, 在离施加电源越远的位置其受力越 小, 阳离子迁移量亦逐渐减小, 导致 IPMC 末端的迁
移量最小, 大大降低其输出性能. 因此有必要减小 IPMC 的表面电阻, 降低电压梯度, 从而增加阳离子 迁移量, 提高 IPMC 的驱动性能. 当铂氨复合物浓度 由 $2 \mathrm{mg} / \mathrm{mL}$ 增加至 $3.5 \mathrm{mg} / \mathrm{mL}$ 时, 镀层厚度由 $6 \mu \mathrm{m}$ 增加为 $24 \mu \mathrm{m}$ (图 5), 表面电阻由 $7.8 \Omega / \mathrm{cm}$ 减小为 1 $\Omega / \mathrm{cm}$, 降低了约 7.8 倍. 另一方面, 致密的较厚的金 属电极具有颗粒密封效应, 有助于减缓水分子的散 失，提高了 IPMC 的驱动效率. Onishi 等 ${ }^{[18]}$ 和 Lee 等 ${ }^{[19]}$ 亦报道通过增加 IPMC 表面金属镀层的厚度以减小 表面电阻，其镀层厚度约为 $20 \mu \mathrm{m}$, 但他们是通过多 次化学镀 (8 次)来实现的, IPMC 的制备工艺过程比较 繁琐和耗时. 本文通过正交实验设计法确定出了合 理的主镀铂氨复合物浓度 $(3.5 \mathrm{mg} / \mathrm{mL})$, 仅采用 2 次 化学镀即获得了 24 $\mu \mathrm{m}$ 厚的铂金属镀层(图 5(d)), 优 化了 IPMC 的制备工艺，一方面降低了 IPMC 的表面 电阻 $(\sim 1 \Omega / \mathrm{cm})$, 减小了电能损耗，同等条件下作用 在 IPMC 两侧的电场强度增加, 膜内单个阳离子所受 电场力增大, 可有效提高 IPMC 的力输出数值; 另一 方面由于较好的颗粒密封效应阻碍了水分子的泄漏, 延长了 IPMC 的离水有效工作寿命. 制备复合金属电 
极也可以减小表面电阻, 例如 Shahinpoor 等人 ${ }^{[7]}$ 利用 电化学沉积了一层薄薄的银(厚 $1 \sim 2 \mu \mathrm{m}$ ) 以减小表面 电阻, Lee 等人 ${ }^{[8]}$ 通过物理气相沉积(PVD)在铂层表面 形成了柔软的金涂层(厚 $1 \sim 2 \mu \mathrm{m}$ ). 本文重点讨论了 铂氨复合物浓度在降低表面电阻和形成优良金属电 极中的作用.

\section{2 水合程度}

IPMC 的电致变形机理源于阳离子的电泳现象, 由于基体 Nafion 膜是全氟磺酸阳离子交换薄膜, 在 外加电场作用下，基体膜中的阳离子与水分子结合 在一起迁移到阴极, 导致阳极含水量减少, 阴极含水 量增加, 使得 IPMC 向阳极偏转, 在交流电压的作用 下 IPMC 会发生摆动, 因此 IPMC 复合材料的弯曲性 能与阳离子的水合程度有很大关系 ${ }^{[7,24]}$. 薄膜内阳离 子水合程度较高, IPMC 会产生较大的输出力和位移. 在 IPMC 驱动过程中, 由于水的电解和蒸发, 薄膜内 含水量降低, 导致水合程度降低, 同时全氟磺酸薄膜 内部固有的离子管道缩小, 严重阻碍了离子的迁移. 因此较高的含水量是保证 IPMC 性能稳定的关键因 素. Lee 等人 ${ }^{[9]}$ 利用聚吡咯/氧化铝复合物的吸湿性和 氧化还原反应来增加含水量和离子迁移, 从而提高 IPMC 的输出性能, 其最大输出力较空白基体膜提高 了 1 倍. 由于离子液有较高的稳定性, 可在较高的电 压下驱动, 许多研究采用离子液代替水分子 ${ }^{[25,26]}$, 如 Bennett 等人 ${ }^{[26]}$ 使用 1-乙基-3-甲基咪唑三氟甲磺酸盐 作为溶剂, 可在离水工作环境下摆动 $7 \times 10^{6}$ 次, 稳定 性能得到了提高, 但是响应频率非常低 $(<0.05 \mathrm{~Hz})$, 限制了 IPMC 的应用领域. 本文通过添加 TEOS 制备 了高储水能力的全氟磺酸离子交换聚合物薄膜, 该 膜内生成了不同尺寸的微管道(图 6(b) (d)), 提高了 含水量(图 7), 进而提高了水合程度. 当 TEOS 的含量 为 $1.5 \%$ 时, Nafion 膜内的微管道直径 $(10 \mu \mathrm{m})$ 和含水 量 $(15.27 \%)$ 为最大, 水合程度最高, 在外加电场作用 下其参与迁移的水合阳离子最多, 优化后 IPMC 的力 输出数值和稳定工作性能均大幅提高. 今后的工作 可以考虑将离子液和水分子结合在一起作为溶剂来 提高水合程度.

\section{3 输出性能}

众多学者为提高 IPMC 的力输出和离水工作寿 命进行了大量的研究. 例如 Lee 等人 ${ }^{[9]}$ 通过添加聚吡
咯/氧化铝复合物来提高 $\operatorname{IPMC}($ 长 $\times$ 宽 $\times$ 高, $20 \mathrm{~mm} \times$ $5 \mathrm{~mm} \times 0.453 \mathrm{~mm}$ )的输出性能, 电压为 $3 \mathrm{~V} \mathrm{DC}$, 单位 厚度下其最大力输出为 $18 \mathrm{mN} / \mathrm{mm}$. Nguyen 等人 ${ }^{[27]}$ 使用蒙脱石和二氧化硅纳米材料作为添加剂制备 $\operatorname{IPMC}(20 \mathrm{~mm} \times 5 \mathrm{~mm} \times 0.3 \mathrm{~mm})$, 电压为 $3 \mathrm{~V} \mathrm{DC}$, 单 位厚度下的最大输出力为 $52 \mathrm{mN} / \mathrm{mm}$. Kim 和 Shahinpoor 等人 ${ }^{[17]}$ 采用聚合物溶液浇铸技术制备了厚 的全氟磺酸薄膜以增加 IPMC $(20 \mathrm{~mm} \times 5 \mathrm{~mm} \times 2 \mathrm{~mm})$ 的输出力, 方波电压 $6 \mathrm{~V} \mathrm{AC}$, 频率为 $0.5 \mathrm{~Hz}$, 其单位厚 度下最大力输出约为 $100 \mathrm{mN} / \mathrm{mm}$. 本文优化后 IPMC 试样的尺寸为 $20 \mathrm{~mm} \times 5 \mathrm{~mm} \times 0.42 \mathrm{~mm}$, 正弦电压 3 $\mathrm{V} \mathrm{AC}$, 频率为 $0.1 \mathrm{~Hz}, \mathrm{IPMC}$ 末端最大输出力为 49.98 $\mathrm{mN}$ (图 4(a)), 其单位厚度下力输出为 $119 \mathrm{mN} / \mathrm{mm}$. 一般情况下 IPMC 的输出力随厚度和激励电压的增 加而增加 ${ }^{[8,14]}$, 上述 IPMC 试样的长宽相同, 但厚度 不同. 通过对单位厚度下 IPMC 输出力的比较, 可见 本文优化后 IPMC 试样的最大输出力密度最大, 获得 了富有竞争力的提升. 在离水工作环境下, Kim 等 ${ }^{[28]}$ 研究了 IPMC 的稳定工作性能, 规定输出位移衰减至 最大值的 $60 \%$ 为离水有效工作寿命，在 $200 \mathrm{~s}$ 左右位 移衰减为 $60 \%$. 而本文优化后的 IPMC 试样的输出位 移在 $316.8 \mathrm{~s}$ 达到最大值的 $80 \%$, 在 $396 \mathrm{~s}$ 达到最大值 的 60\%(图 3(c)), 较 Kim 的研究离水有效工作寿命延 长了近 1 倍, 稳定工作能力亦有可观的提升. 其原因在 于本文通过选择合理的铂氨复合物浓度获得了优良 的金属电极层，添加定量的 TEOS 大幅提高了储水能 力和水合程度, 综合这两个方面对 IPMC 进行了优化. 因此较国际同行制备的 IPMC 材料, 本文优化后“人 工肌肉”的最大输出力和稳定工作能力均获得提高.

\section{4 结论}

本文采用正交实验设计法, 拟定 3 个影响因子, 分别为还原剂浓度, 铂氨复合物浓度, TEOS 含量, 选取 $\mathrm{L}_{16}\left(4^{5}\right)$ 正交表设计实验方案, 优化了 IPMC 的制 备工艺, 提高了 IPMC 的输出力和离水工作寿命, 使 得 IPMC 在水下微型机器人驱动、微机电系统和“人 工肌肉”等领域具有较好的应用。

(1) 实验结果的分析表明: 在拟定的 3 个影响因 子中, 主镀铂氨复合物的浓度对 IPMC 输出力有高度 显著的影响, Nafion 膜中 TEOS 的含量对 IPMC 输出 力有显著的影响，还原剂的浓度对 IPMC 输出力没有 明显的影响. 
(2) 在最优条件 $\mathrm{A}_{2} \mathrm{~B}_{4} \mathrm{C}_{4}$ 下, 即还原剂浓度为 $1.5 \%$, 铂氨复合物浓度为 $3.5 \mathrm{mg} / \mathrm{mL}$, TEOS 含量为 $1.5 \%$, 制备得 IPMC 的最大输出力为 $50 \mathrm{mN}$, 相应的 最大输出位移为 $14.10 \mathrm{~mm}$. 较常规条件, 在最大输
出位移性能相当的情况下, 优化条件下 IPMC 的最大 力输出提高了 2.4 倍, 输出力有效工作寿命延长了 5.8 倍, 输出位移有效工作寿命延长了 5 倍, 为 IPMC 进一步的应用研究提供了基础保障.

\section{参考文献}

1 国家自然科学基金委员会工程与材料科学学部.机械工程学科发展战略报告(2011-2020). 北京：科学出版社, 2010

2 Aliev A E, Oh J, Kozlov M E, et al. Giant-stroke, superelastic carbon nanotube aerogel muscles. Science, 2009, 323: 1575-1578

3 Lehmann W, Skupin H, Tolksdorf C, et al. Giant lateral electrostriction in ferroelectric liquid-crystalline elastomers. Nature, 2001, 410: $447-450$

4 Liu S, Liu Y, Cebeci H, et al. High electromechanical response of ionic polymer actuators with controlled-morphology aligned carbon nanotube/Nafion Nanocomposite electrodes. Adv Funct Mater, 2010, 20: 3266-3271

5 Bar-Cohen Y. Electroactive Polymer (EAP) Actuators as Artificial Muscles: Reality, Potential, and Challenges. Washington: SPIE Press, 2001

6 Kim K J, Shahinpoor M. Ionic polymer-metal composites: II. Manufacturing techniques. Smart Mater Struct, 2003, 12: 65-79

7 Shahinpoor M, Kim K J. The effect of surface electrode resistance on the performance of ionic polymer-metal composite (IPMC) artificial muscles. Smart Mater Struc, 2000, 9: 543-551

8 Lee S G, Park H C, Pandita S D, et al. Performance improvement of IPMC (ionic polymer metal composites) for a flapping actuator. Int J Con Aut Sys, 2006, 4: 748-755

9 Lee J W, Kim J H, Chun Y S, et al. The performance of Nafion-based IPMC actuators containing polypyrrole/alumina composite fillers. Macromol Res, 2009, 17: 1032-1038

10 Shahinpoor M, Kim K J. Ionic polymer-metal composites: IV. Industrial and medical appilications. Smart Mater Struc, 2005, 14: 197-214

11 Park I S, Kim S M, Pugal D, et al. Visualization of the cation migration in ionic polymer-metal composite under an electric field. Appl Phys Lett, 2010, 96: 0433011-0433013

12 Shoji E, Hirayama D. The effects of the potential and polarization time on the performance of ionic polymer metal composite actuators: A control of forward and reverse displacements. Polym Adv Technol, 2007, 18: 346-352

13 Tiwari R, Kim K J. Disc-shaped ionic polymer metal composites for use in mechano-electrical applications. Smart Mater Struct, 2010, 19: 0650161-0650167

14 Yu M, Shen H, Dai Z D. Manufacture and performance of ionic polymer-metal composites. J Bionic Eng, 2007, 4: 143-149

15 Zhang W, Guo S X, Asaka K. A new type of hybrid fish-like microrobot. Int J Autom Comp, 2006, 4: 358-365

16 Yeom S W, Oh I K. A biomimetic jellyfish robot based on ionic polymer metal composite actuators. Smart Mater Struct, 2009,18: 085022

17 Kim K J, Shahinpoor M. A novel method manufacturing three-dimensional ionic polymer-metal composites (IPMC) biomimetic sensors, actuators and artificial muscles. Polymer, 2002, 43: 797-802

18 Onishi K, Sewa S, Asaka K, et al. Morphology of electrodes and bending response of the polymer electrolyte actuator. Electrochim Acta, 2000, 46: 737-743

19 Lee S J, Han M J, Kim S J, et al. A new fabrication method for IPMC actuators and application to artificial fingers. Smart Mater Struct, 2006, 15: 1217-1224

20 Lee D Y, Park I S, Lee M H, et al. Ionic polymer-metal composite bending actuator loaded with multi-walled carbon nanotubes. Sens Actuators A, 2007, 133: 117-127

21 Guo D J, Ding H T, Wei H J, et al. Hybrids perfluorosulfonic acid ionomer and silicon oxide membrane for application in ion-exchange polymer-metal composite actuators. Sci China Ser E: Technol Sci, 2009, 52: 3061-3070

22 Millet P. Preparation of solid polymer electrolyte composites: investigation of the ion-exchange process. J Appl Elec, 1995, 25: 227-232

23 Millet P. Preparation of solid polymer electrolyte composites: investigation of the precipitation process. J Appl Elec, 1995, 25: 233-239

24 Lee J H, Lee J H, Nam J D, et al. Water uptake and migration effects of electroactive ion-exchange polymer metal composite (IPMC) actuator. Sens Actuators A, 2005, 118: 98-106

25 Lee J W, Yoo Y T. Anion effects in imidazolium ionic liquids on the performance of IPMCs. Sens Actuators B, 2009, 137: 539-546

26 Bennett M D, Leo D J. Ionic liquids as stable solvents for ionic polymer transducers. Sens Actuators A, 2004, 115: 79-90

27 Nguyen V K, Lee J W, Yoo Y T. Characteristics and performance of ionic polymer-metal composite actuators based on Nafion/layered silicate and Nafion/silica nanocomposites. Sens Actuaotrs B, 2007, 120: 529-537

28 Kim S J, Lee I T, Kim Y H. Performance enhancement of IPMC actuator by plasma surface treatment. Smart Mater Struct, 2007, 16: N6-N11 